\title{
CD133 and CD24 expression in renal tissue of patients affected by autosomal dominant polcystic kidney disease
}

\author{
Daniele Lodi, Giulia Ligabue, Fabrizio Cavazzini, Valentina Lupo, Gianni Cappelli, \\ Riccardo Magistroni ${ }^{*}$ \\ Department of Medicine and Medical Specialties, Division of Nephrology Dialysis and Transplantation, University of Modena and

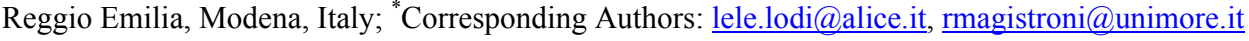

Received 12 February 2013; revised 12 March 2013; accepted 21 March 2013

Copyright (C) 2013 Daniele Lodi et al. This is an open access article distributed under the Creative Commons Attribution License, which permits unrestricted use, distribution, and reproduction in any medium, provided the original work is properly cited.

\section{ABSTRACT}

Background: Autosomal dominant polycystic kidney disease is a condition mainly characterized by the progressive development and enlargement of cysts in each kidney. In this process a high rate of proliferation and apoptosis of tubular cells has been documented and interpreted as a futile attempt of tissue repair. In consideration of the role of stem cells in reparative processes we investigated the presence and localization of $\mathrm{CD} 133+\mathrm{CD} 24+$ renal progenitors in renal ADPKD tissue and cells. Methods: Two normal kidneys and two ADPKD kidneys were examined. CD133 and CD24 expression was investigated by confocal microscopy and immunoblotting. Furthermore cystic isolated cells and cultured immortalized cells were characterized. Results: CD133 and CD24 have the same localization in ADPKD tissues and in normal kidneys: expression is restricted to a subset of epithelial cells (PEC) of Bowman's capsule and to tubular cells in a focal and segmental pattern. Furthermore, in ADPKD tissue, cysts diffusely express CD133 and CD24. According to a quantitative analysis in ADPKD tissue CD133 + CD24 + cells are statistically more expressed in tubules $(p<$ 0.001 ) and less expressed in the Bowman's capsule $(p=0.0016)$ compared to the same localizations in control tissue. Conclusions: CD133 and CD24 antigens, typically expressed by renal epithelial progenitors, are more expressed in ADPKD tubules and highly expressed in ADPKD cysts. Whether CD133 and CD24 expression would signify renal progenitor recruitment or alternatively an expression pattern of the dedif- ferentiation of ADPKD cells remains unclear.

Keywords: Renal Stem Cells; CD133; CD24; ADPKD

\section{INTRODUCTION}

Autosomal Dominant Polycystic Kidney Disease (ADPKD) can arise from mutations in either PKD1 (85\% of affected patients) or PKD2 (15\% of affected patients) genes with clinically indistinguishable manifestations [1]. ADPKD affects over 1:1000 of the worldwide population and it causes 1:3000 hospitalization. ADPKD is asymptomatic in about $50 \%$ of subjects with pkd genes mutated [2]. It is a systemic disease associated with renal failure, intracranial arterial aneurysms, cardiac valvular defects, colonic diverticulosis and cyst formation in other organs such as the liver, spleen and pancreas. Nevertheless ADPKD gives mainly renal manifestations: the patients develop renal cysts which progressively lead to disruption of renal parenchyma, interstitial fibrosis, cellular infiltration and loss of functional nephrons. Renal degeneration finally progresses to end-stage renal disease [1].

PKD1 (located on chromosome 16) codes for a large transmembrane protein of $460 \mathrm{KDa}$ involved in the control of calcium permeation and cell-cell/matrix interaction. PKD2 (located on chromosome 4) codes for a calcium channel of about $110 \mathrm{KDa}$. The polycystin 1 and 2 (the proteins were coded by PKD1 and PKD2 genes) collaborate together in a functional complex $[3,4]$.

Previous studies have demonstrated a high rate of proliferation and apoptosis in the cysts of ADPKD patients4 and this phenomenon has been interpreted as an unproductive attempt of tissue reparation. In this scenario a 
likely involvement of renal progenitors can be hypothesized.

Sagrinati et al. identified, in human adult kidneys, a subset of Parietal Epithelial Cells (PECs) in the Bowman's capsule that revealed self-renewal potential and high cloning efficiency. This subset of PECs exhibits coexpression of stem cell markers CD24 and CD133 and absence of lineage-specific markers. Under appropriate culture conditions, individual clones of CD24 + CD133+ PEC could be induced to generate mature, functional, tubular cells with phenotypic features of proximal and/or distal tubules [5].

ADPKD is characterized by expansion of cysts and tubular hyperplasia in the context of a sustained proliferative and apoptotic process. In this brief report we explored the pathologic features of presumptive renal progenitor cells in ADPKD renal tissue trying to explain the nature of their involvement in the disease: tissue repair or increase of cystic lesions.

\section{RESULTS}

\subsection{Expression of CD133 and CD24 in Normal and ADPKD Kidney}

In normal kidney at the glomerular level the confocal microscopic analysis shows that CD133 and CD24 are co-expressed by a subset of parietal epithelial cells (PEC) in the Bowman's capsule (Figure 1: on line publication): CD24 has a generalized and diffuse presentation, CD133 is generalized and segmental. At tubular level the distribution of the expression of both CD133 and CD24 is focal and global. In the normal kidney specimen the overall intensity of CD24 + cells have the similar median value compared to CD133 + cells but the distribution of the intensity for the CD24 + cells is larger (Figure 2(a): on line publication). In the ADPKD kidney specimen the overall intensity of CD133 is higher, but not statistically significant, than the intensity of CD24 + cells (Figure 2(b): on line publication). It is interesting to note that at cellular level CD133 is polarized to the luminal side of the tubular cell surface as well to the urinary space side in positive PECs, whereas CD24 is not clearly polarized but shows a diffuse distribution at the membrane and cytoplasm level (Figure 1: on line publication).

Analysis of confocal microscopy of ADPKD kidneys revealed that CD133 and CD24 were expressed in a subset of glomerular (Figures 3(a)-(c)) and tubular (Figures 3(d)-(f)) cells and they showed the same tissue of normal kidney [5]: CD133 presents a generalized and segmental pattern while CD24 is generalized and diffuse in the Bowman's capsule. The expression of both CD133 and CD24 is focal and global in the tubules.

The cysts of ADPKD samples showed a segmental
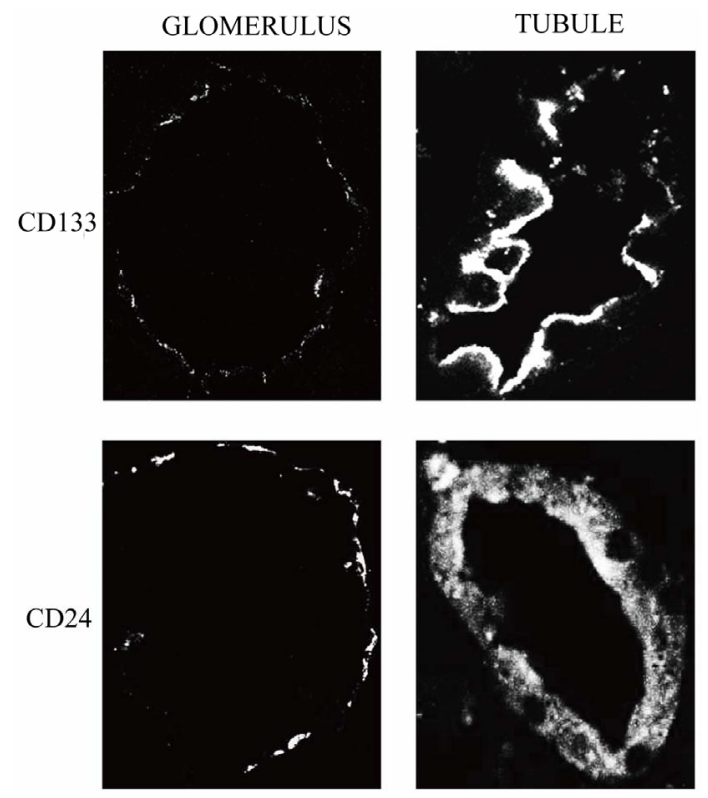

Figure 1. CD133 and CD24 expression in normal kidney. The first lane shows tissue expression of CD133 in Bowman's capsules and tubules. CD133 is expressed in a subset of PEC in the Bowman's capsule and in the luminal side of positive tubular cells. The second lane shows tissue expression of CD24 in Bowman's capsules and tubules. CD24 is expressed in a subset of PEC in the Bowman's capsule and in the cytoplasm of tubular cells.

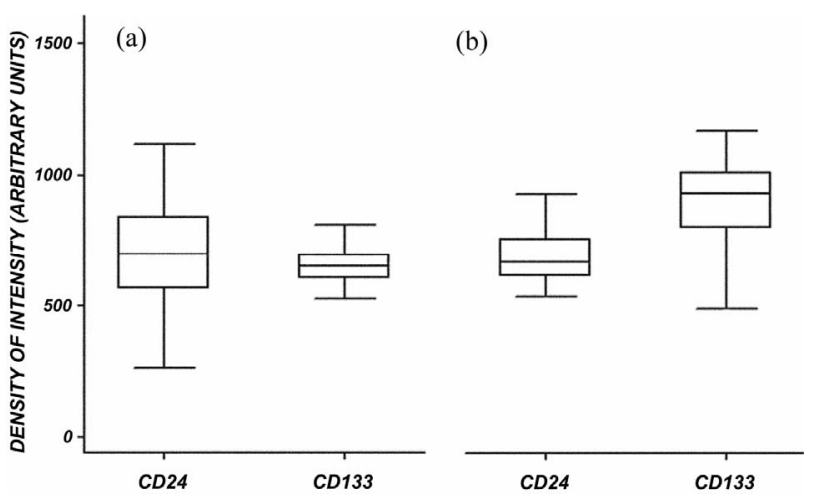

Figure 2. Morphometric analysis of CD24 and CD133 global expression (a) in normal kidney and (b) in ADPKD kidney. (a) In normal tissue, CD24 and CD133 the median expression is similar, even though CD24 intensity has a larger distribution than CD133; (b) In ADPKD tissue, CD133 expression is higher than that of CD24.

co-expression pattern of CD133 and CD24. CD24 has a generalized distribution in the samples while CD133 is segmental. The cells of large cysts show a flatted morphology and a co-localization of CD133 and CD24 can be appreciated in some segments (Figures 3(g)-(i)).

The quantitative comparison between normal kidney and ADPKD kidney revealed an increased CD133 expression in tubules while glomerular expression appears 

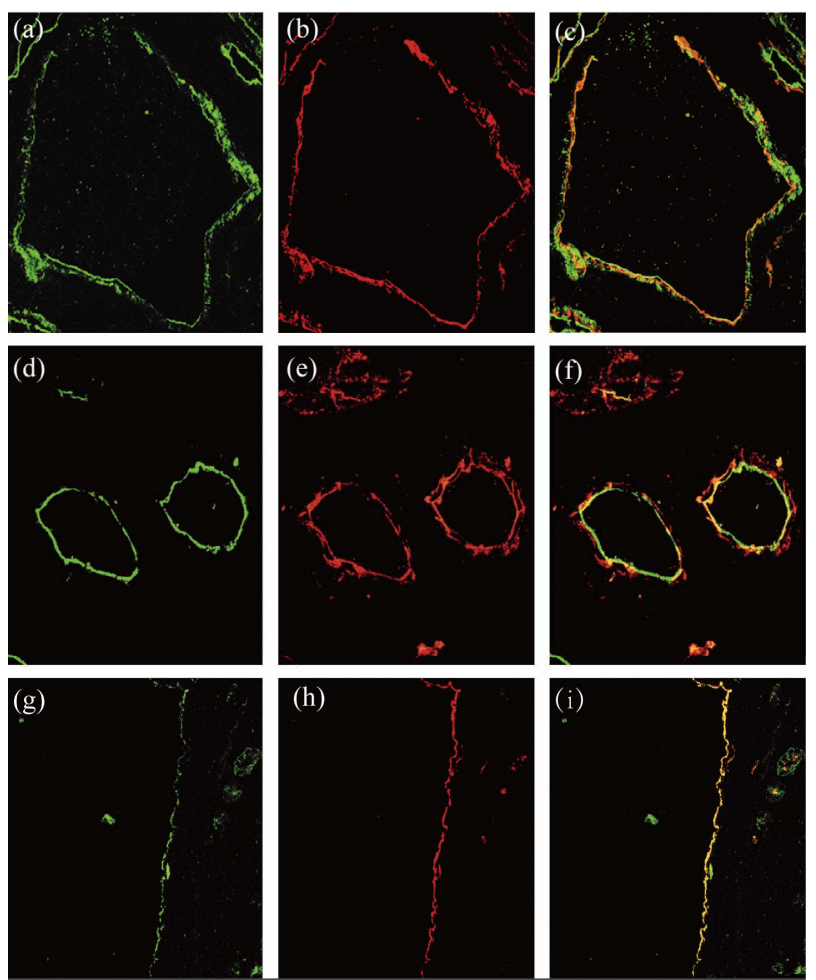

Figure 3. CD133 and CD24 expression in ADPKD samples. The first lane shows glomerular expression of CD133 (a) and CD24 (b). CD133 and CD24 are co-localized and co-expressed by a subset of PEC in the Bowman's capsule (c). The second lane shows tubular expression of CD133 (d) and CD24 (e). CD133 and CD24 are co-expressed in these tubules (f). The third lane shows cystic expression of CD133 (g) and CD24 (h). CD133 and CD24 are co-localized and co-expressed by the cystic cells (i).

reduced in ADPKD samples (Figure 4). After image analysis Mann-Whitney test resulted statistically significant for these comparisons (ADPKD tubules vs Normal tubules: $\mathrm{z}=-7.681 \mathrm{p}<0.001$; ADPKD Glomerula vs Normal Glomerula: $z=3.162 \mathrm{p}=0.0016)$. CD 24 expression in glomerular and tubules does not show quantitative difference between normal and ADPKD samples (Figure 4).

\subsection{Expression of CD133 and CD24 in Cultured Cells}

Cells deriving from the cystic wall of ADPKD tissue were scraped and expanded in culture. The epithelial nature of these cells was confirmed by cytocheratin immunohistochemical characterization (Figure 5 and Table 1: on line publication). In the subsequent analyses were used the clones of isolated cells that showed the complete expression of the cytokeratins (CAM 5.2, CK 7, MNF 116) usually expressed by cystic cells in tissue specimens.

Isolated cystic cells show a characteristic expression

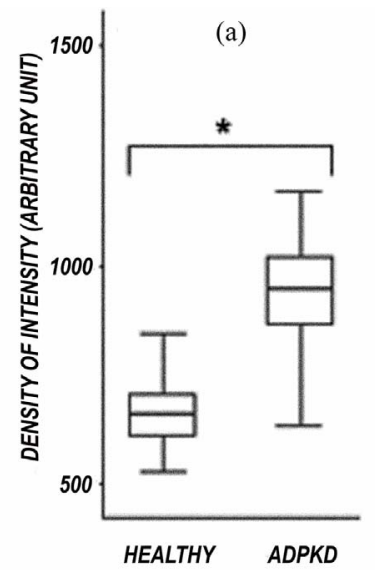

(c)

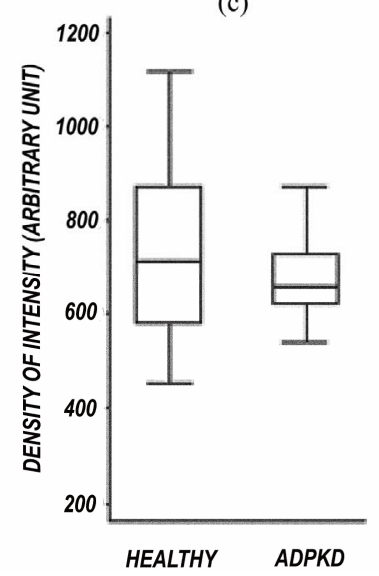

(b)

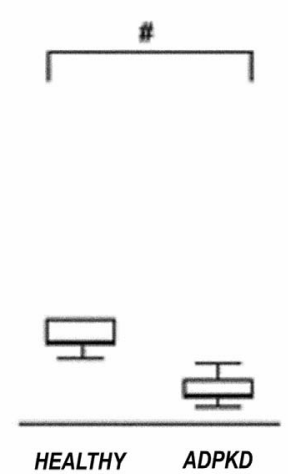

(d)

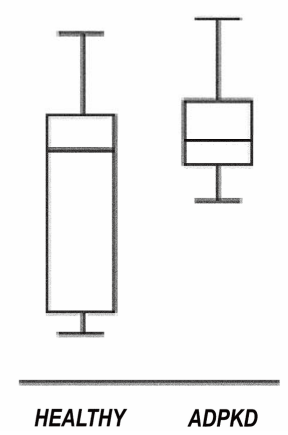

Figure 4. Morphometric analysis of CD133 and CD24 expression in tubules and glomerula of normal kidney and ADPKD kidney. CD133 expression is higher in ADPKD tubules than in normal tubules $\left({ }^{*} \mathrm{p}<0.001\right)$. (a) CD133 expression is reduced in ADPKD glomerula compared to normal glomerula $\left({ }^{\#} \mathrm{p}=\right.$ 0.0016); (b) CD24 expression is not statistically different in both normal tubules vs ADPKD tubules (c) and normal glomerula vs ADPKD glomerula (d) comparison.
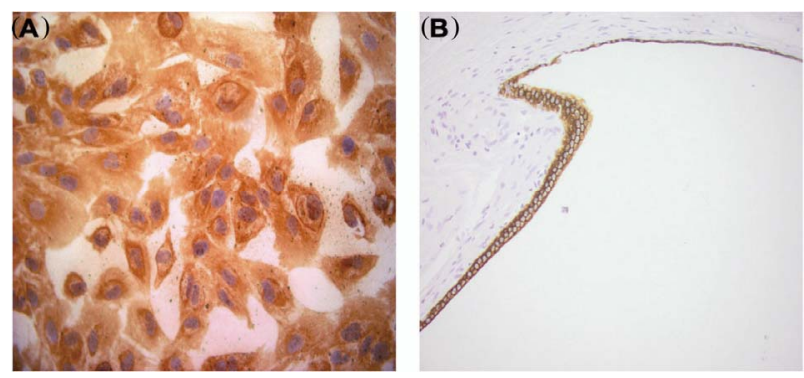

Figure 5. Example of cytokeratin expression (CK 7) in cultured cystic cells and in cyst. Immunohistochemical analysis reveals that both cultured cystic cells (A) and cystic epithelium (B) express CK 7.

pattern for CD133 and CD24 (Figures 6(A)-(C): on line publication). CD133 is localized on the cellular membrane (Figure 6(A): on line publication) while CD24 is globally diffused in the cytoplasm (Figure 6(B): on line 
Table 1. Immunohistochemical analysis of cytokeratin expression in ADPKD tissue. (1) Focal and global distribution; (2) Focal and segmental distribution.

\begin{tabular}{ccccc}
\hline & CAM 5.2 & CK 7 & CK 20 & MNF 116 \\
\hline Bowman's Capsule & - & $+(2)$ & - & $+(2)$ \\
Tubules & $+(1)$ & $+(1)$ & - & $+(1)$ \\
Cysts & $+(2)$ & $+(2)$ & - & $+(2)$ \\
\hline
\end{tabular}
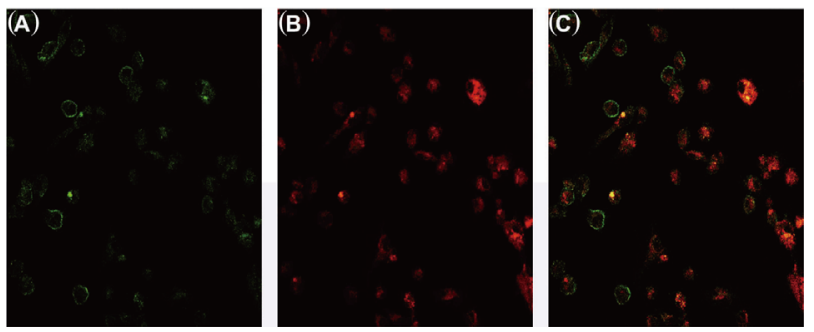

Figure 6. CD133 and CD24 expression in cystic cells in colture. CD133 is closely expressed by the cellular membrane (A). CD24 is expressed in the cytoplasm (B). CD133 and CD24 are co-expressed by the cystic cells $(\mathrm{C})$.

publication). The expression of CD24 is more diffuse while few cells are CD133+; only a small subset of isolated cystic cells are both CD133 and CD24 positive.

The same expression pattern is appreciable in HK-2 cell line while in HEK-293 cell line this peculiar pattern is lacking, both antigens presenting a cytoplasmic distribution (Figure 7: on line publication).

By western blot analysis CD133 is highly expressed by cystic cells, HK-2 presents an intermediate level of expression while HEK-293 cell line shows an almost absent expression (Figure 8). Analogous amounts of CD24 can be detected in the three cellular populations (Figure 8).

\section{DISCUSSION}

The high rate of proliferation and apoptosis of cystic cells is a well known phenomenon in ADPKD [4]. It has been interpreted as an unsuccessful proliferative repair response of abnormal tubular cells degenerating into cyst formation. The abnormal behavior of the tubule in ADPKD patients origins from genetic ablation of a critical function of the polycystin proteins and primary cilia $[6,7]$. This system is supposed to act as a sensor for renal injury monitoring the urinary intratubular flux. According to this model an abnormal constitutive activation of this pathway in ADPKD leads to the futile (proliferative) attempt to repair an essentially non existing injury [4].

In this scenario where proliferation and renal tissue repair are active we could speculate a possible role for renal progenitors. The target of our report has been an explorative analysis of surface antigens related to stemness in ADPKD tissue. A multipotent progenitor cell
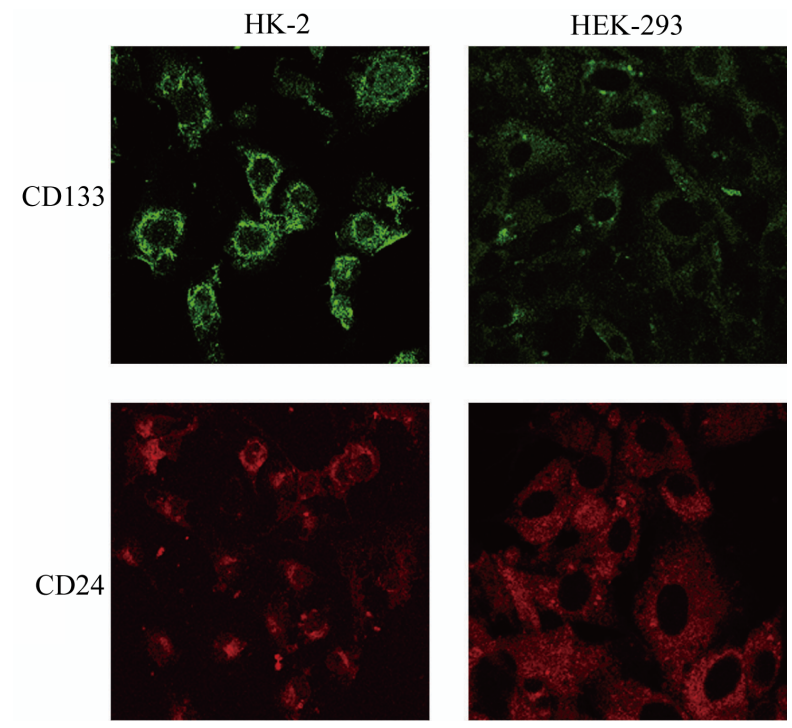

Figure 7. CD133 and CD24 expression in cellular lines. HK2 show a similar antigen-expression pattern to cystic cells: CD133 is localized on the cellular membrane and CD24 is localized in the cytoplasm. HEK-293 have a diffused and low cellular expression of CD133 and CD24.

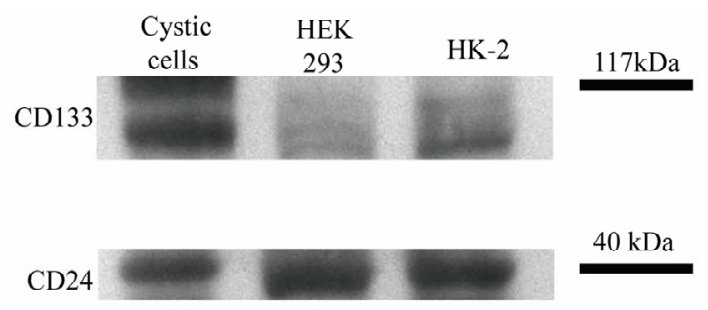

Figure 8. Western blot analysis of CD133 and CD24 expression in untreated HEK-293, HK-2 and cystic cells. Cystic cells express the highest amount of CD133 whereas HEK-293 the lowest. CD24 amount is similar in the three populations.

population constituted by $\mathrm{CD} 24+$ and $\mathrm{CD} 133+$ parietal epithelial cells (PECs), in adult human glomerula has been described. In this report a murine model of acute renal failure caused by glycerol induced rhabdomiolysis has been generated and injection of CD24+ and CD133+ PECs differentiated into tubular cells significantly ameliorating kidney damage 5. CD133 is a pentaspan transmembrane glycoprotein suspected to have a role in maintaining stem cell properties by suppressing differentiation and promoting proliferation. CD133 expression is also associated to several types of cancer [8-10]. CD133 function is not clear but its presence on early and undifferentiated cells is suggestive of a growth factor receptor, and the presence of five tyrosine residues on the 50-aa cytoplasmic tail may indicate that the protein is phosphorylated in response to ligand binding and triggers a signal transduction [11]. CD24 is a sialoglycoprotein expressed on mature granulocytes [12], B and T lymphocyte subsets $[13,14]$, normal and cancer stem cells 
$[15,16]$. The protein is anchored via a glycosyl phosphatidylinositol to cell surface. It acts as adhesion molecule and is involved in cell migration and signal transduction.

In our specimens we could appreciate the presence of a rich CD24+ and CD 133+ cell population. In ADPKD the distribution of these cells is qualitatively similar to normal tissue with the notable exception of a significant expression in cystic cells. Quantitatively CD 133+ cell population appears globally increased in ADPKD tissue. In particular we found a statistically significant increase of CD133 expression in tubules while glomerular expression appears reduced in ADPKD compared to control tissue. In this scenario the speculative hypothesis of an active migration of progenitors from the glomerular "niche" [17] to the tubule interstitial compartment is intriguing.

The abundant presence of CD133+ and CD24+ cells in ADPKD renal tissue sustains the hypothesis of an important role of this cell population in the disease. Inflammation and tubular damage are common features of ADPKD and CD133+ and CD24+ cells could likely play a role in the reparative process. Furthermore the diffuse presence of these elements in the cystic wall suggest that they could contribute to cystic expansion and disease progression. Anyway as far as now no evidence is available to sustain the hypothesis that CD133+ and CD24+ cystic cells are progenitors migrated into the cystic wall rather than the alternative hypothesis that they represent dedifferentiated [18] tubular cells that express "de novo" CD133 and CD24.

The expression of CD133 and CD24 is maintained in cystic cells isolated and cultured in vitro. Furthermore we evaluated the expression of these antigens in two tubular immortalized cellular lines, HEK293 and HK2.

\section{CONCLUSION}

In conclusion we demonstrated an abundant population of $\mathrm{CD} 133+\mathrm{CD} 24+$ cells in renal tissue obtained from ADPKD patients. This cell population is present in the cystic wall and abundant in tubules, which conversely seems to be reduced in ADPKD glomerula in comparison to normal tissue. The role of this cells was not clear yet, but it could be interesting to evaluate more cases of ADPKD, in different stages (from asymptomatic to late stage), and clarify if CD133+ CD24+cells were prognostic factors for a poorer or better evolution of the disease. Additionally, we cannot univocally conclude that the CD133 + CD24 + cystic cells represent migrated renal progenitors rather than tubular dedifferentiated cells 18 , but with a deeper biomarker analysis we could understand their origin and their hypothetical migration. Knowing this element will be important to understand more in detail pathophysiology of ADPKD and find new target to delay the progression of the disease.

\section{MATERIAL AND METHODS}

\subsection{Antibodies}

CD133 monoclonal (293C3) antibody for immunofluorescence was provided by Miltenyi Biotec (Miltenyi Biotec, Bergisch Gladbach, Germany). CD133 monoclonal (W6B9C1) antibody for Western blot was provided by Miltenyi Biotec (Miltenyi Biotec, Bergisch Gladbach, Germany). CD24 monoclonal (SN3) antibody was provided by Santa Cruz (Santa Cruz Biotechnology Inc., Santa Cruz CA, USA). PKD1 rabbit polyclonal (H260) antibody was provided by Santa Cruz (Santa Cruz Biotechnology Inc., Santa Cruz CA, USA). Goat anti mouse IgG2b-Alexa 568 conjugate, goat anti mouse IgG2b-Alexa 633 conjugate, goat anti mouse IgG1-Alexa 568 conjugate, goat anti rabbit IgG-Rhod conjugate, goat anti mouse IgG-HRP conjugate were used as secondary antibodies. Cytokeratins monoclonal (CAM 5.2, CK7 [RCK105], CK20 [SPM140] and MNF 116) antibody was provided by Becton Dickinson (only CAM 5.2) (Becton Dickinson, CA, USA) and Abcam (the others) (Abcam, Cambridge, UK).

\subsection{Tissues}

Normal kidney fragments were obtained from a cadaveric kidney donor. The kidney was not suitable for transplantation due to the incidental discovery of a neoplastic nodule of $5 \mathrm{~cm}$ at the lower pole upon macroscopic examination. The pathologic examination diagnosed a clear cell carcinoma (grade 2 according to Fuhrman classification; T1bN0M0 according to the International Union Against Cancer classification); the lower pole was excised and the remaining parenchyma was used in the following procedures. The remaining parenchyma did not reveal any evidence of clear cell carcinoma.

Polycystic kidney tissue was obtained from 2 ADPKD patients underwent to nephrectomy in preparation for receiving renal transplantation. All procedures were preceded by the approval of our Local Ethical Committee on human experimentation and informed consent was obtained from the patients.

\subsection{Culture}

Human Kidney 2 cells (HK-2) were maintained in DMEM F-12 (Invitrogen/GIBCO, Cralsbad, California) supplementated with 10\% FBS (PAA laboratories $\mathrm{GmbH}$, Pasching, Austria), $2000 \mathrm{U} / \mathrm{ml}$ penicillin (PAA laboratories $\mathrm{GmbH}$, Pasching, Austria), $2000 \mathrm{ug} / \mathrm{ml}$ streptomycin (PAA laboratories $\mathrm{GmbH}$, Pasching, Austria), and $20 \mathrm{mM}$ L-glutamine (Invitrogen/GIBCO, Cralsbad, California).

Human Embryonic Kidney 293 cells (HEK-293) were maintained in DMEM (Invitrogen/GIBCO, Cralsbad, 
California) supplementated with 5\% FBS (PAA laboratories $\mathrm{GmbH}$, Pasching, Austria), $2000 \mathrm{U} / \mathrm{ml}$ penicillin (PAA laboratories GmbH, Pasching, Austria), 2000 ug/ml streptomycin (PAA laboratories $\mathrm{GmbH}$, Pasching, Austria), and $20 \mathrm{mM}$ L-glutamine (Invitrogen/GIBCO, Cralsbad, California).

HK-2 and HEK-293 were grown two weeks and medium was changed every two days. After every week, cells were washed with PBS (PAA laboratories $\mathrm{GmbH}$, Pasching, Austria) and were trypsinizated (Invitrogen/ GIBCO, Cralsbad, California). Cells harvested were, in part, seeded on chambers slides and the others were used to extract proteins.

Cystic cells were obtained by fragments of renal cysts deprived of external membrane. Each cyst was picked up, was divided in some little fragments and were digested by collagenase (Invitrogen/GIBCO, Cralsbad, California) for one hours. Every pieces of a single cysts were seeded on a plate, previously treated by collagene type I (Invitrogen/GIBCO, Cralsbad, California), the same medium used for $\mathrm{HK}-2$.

\subsection{Immunofluorescence}

Three-micrometer frozen kidney samples mounted on Super Frost Plus slides (Menzel-Gläser, Thermo Scientific, Waltham, MA) were cut by a cryostat (Leica 1720, Leica Mycrosystems, Heerbrog, Germany). The sections, or cellular spots, were fixed with PFA for $10 \mathrm{~min}$, washed with PBS. Slides were coated (3\% Bovine Serum Albumin in PBS buffer) for $1 \mathrm{~h}$ at $20^{\circ} \mathrm{C}$. Slides were incubated by primary antibody for $1 \mathrm{~h}$ at $37^{\circ} \mathrm{C}$, washed with PBS and successively incubated by secondary antibody for $1 \mathrm{~h}$ at $20^{\circ} \mathrm{C}$. Primary and secondary antibodies were diluted in coating solution. Slides were closed with Vectashield $^{\circledR}$ Mounting Medium with DAPI (Vector Laboratories, Inc., Burlingame, CA, USA). Tissue samples, or cells, were examined by immunofluorescence microscopy (Olympus BX41 Microscopy). Laser Confocal images were obtained with Leica TCS SP2 (Leica Microsystems, Heerbrog, Germany).

\subsection{Immunohistochemical}

The same type of sample mounted on slides used for immunofluorescence analysis was handled for immunohistochemical evaluation. Slides were coated (3\% Bovine Serum Albumin in PBS buffer) for $1 \mathrm{~h}$ at $20^{\circ} \mathrm{C}$. Slides were incubated by primary antibody over night at $4^{\circ} \mathrm{C}$, washed with PBS and successively incubated by secondary antibody (conjugated horse radish peroxidise) by for $30 \mathrm{~min}$ at $20^{\circ} \mathrm{C}$. Hydrogen peroxide and diaminobenzidine (DAB) (SIGMA-ALDRICH, St. Louis, MO, USA) were used to obtain chromogen reaction. Olympus BX41 microscope was used to evaluate the samples.

\subsection{Image Analysis}

Images of immunofluorescence-marked kidney were sampled by an acquisition system composed by Olympus BX41 microscope, Olympus XC30 and CELL B 3.0 analysis image processing (Olympus Europa $\mathrm{GmbH}$, Hamburg Germany). Images were saved in tagged image file format (.tiff). The files were analyzed with Inspector Matrox (Matrox Electronic System Ltd., Quebec Canada). ROI (region of interest) defines a portion of image that contains areas to analyze. ROI of each renal component (tubules, glomeruli and cysts) were sampled and converted in a list of single grey tone amount, from white (highest value) to black (zero). Background fluorescence was obtained sampling spot of negative structure, such mesangium or negative tubules, in every image.

\subsection{Statistical Analysis}

Total brightness/positive area ratio was calculated for each ROI. The following analysis of expression were performed: CD24+ in tubules of normal tissue, CD24+ in glomeruli of normal tissue, CD24+ in tubules of ADPKD samples, CD24+ in glomeruli of ADPKD samples, CD24+ in cysts of ADPKD samples, CD133+ in tubules of normal samples, CD133 + in glomeruli of normal tissue, CD133+ in tubules of ADPKD samples, CD133 + in glomeruli of ADPKD samples, CD133 + in cysts of ADPKD samples. Normal and ADPKD tissue components were compare by Mann-Whitney test. Results were considered statistically significant for $\mathrm{p}<0.01$. All statistical analysis were performed with the software SPSS Statistics 19.0.1 (IBM corporation, New York, USA).

\subsection{Western Blot Analysis}

Total proteins were extracted from cellular pellets with Triton X-100 based lysis buffer. Proteins were loaded on SDS-PAGE (3\% stacking, 7.5\% resolving), previously denatured with 4X Reducing Loading Buffer $(200 \mathrm{mM}$ TrisHCl, 20\% $\beta$-Mercapto Ethanol, 8\% SDS, 0.4\% Bromofenol Blue, $40 \%$ glycerol). After SDS-PAGE run and transblot, nitrocellulose membranes were previously incubated by antibodies anti-CD133 and anti-CD24 and subsequently by horse radish peroxidise conjugated secondary antibodies. The detection was obtained by exposition of radiological plates to peroxidise substrate activated by the enzyme.

\section{DISCLOSURES}

The authors certify that there is no conflict of interest with any financial organization regarding the material discussed in the manuscript. 


\section{ACKNOWLEDGEMENTS}

This study was supported by the Research Program "Regione-Università 2007-2009-Innovative Research" granted by the Regional Sanitary Service of Emilia Romagna, Italy.

\section{REFERENCES}

[1] Chang, M.Y. and Ong, A.C. (2008) Autosomal dominant polycystic kidney disease: Recent advances in pathogenesis and treatment. Nephron Physiology, 108, 1-7. http://dx.doi.org/10.1159/000112495

[2] Wei, W., Hackmann, K., Xu, H., Germino, G. and Qian, F. (2007) Characterization of cis-autoproteolysis of polycystin-1, the product of human polycystic kidney disease 1 gene. Journal of Biological Chemistry, 282, 2172921737. http://dx.doi.org/10.1074/jbc.M703218200

[3] Delmas, P., Padilla, F., Osorio, N., Coste, B., Raoux, M. and Crest, M. (2004) Polycystins, calcium signaling, and human diseases. Biochemical and Biophysical Research Communications, 322, 1374-1383. http://dx.doi.org/10.1016/j.bbrc.2004.08.044

[4] Weimbs, T. (2007) Polycystic kidney disease and renal injury repair: Common pathways, fluid flow, and the function of polycystin-1. American Journal of Physiology, Renal Physiology, 293, F1423-F1432. http://dx.doi.org/10.1152/ajprenal.00275.2007

[5] Sagrinati, C., Netti, G.S., Mazzinghi, B., et al. (2006) Isolation and characterization of multipotent progenitor cells from the Bowman's capsule of adult human kidneys. Journal of the American Society of Nephrology, 17, 24432456. http://dx.doi.org/10.1681/ASN.2006010089

[6] Rodat-Despoix, L. and Delmas, P. (2009) Ciliar functions in the nephron. Pflugers Archiv, 458, 179-187. http://dx.doi.org/10.1007/s00424-008-0632-0

[7] Praetorius, H.A. and Spring, K.R. (2003) The renal cell primary cilium functions as a flow sensor. Current Opinion in Nephrology and Hypertension, 12, 517-520. http://dx.doi.org/10.1097/00041552-200309000-00006

[8] Tirino, V., Desiderio, V., Paino, F., et al. (2011) Human primary bone sarcomas contain CD133+ cancer stem cells displaying high tumorigenicity in vivo. FASEB Journal, 25, 2022-2030.

[9] Lorico, A. and Rappa, G. (2011) Phenotypic heterogeneity of breast cancer stem cells. Journal of Oncology, 2011,
135039. http://dx.doi.org/10.1155/2011/135039

[10] Janikova, M., Skarda, J., Dziechciarkova, M., et al. (2010) Identification of CD133+/nestin+ putative cancer stem cells in non-small cell lung cancer. Biomedical Papers of the Medical Faculty of the University Palacky, Olomouc, Czechoslovakia, 154, 321-326. http://dx.doi.org/10.5507/bp.2010.048

[11] Miraglia, S., Godfrey, W., Yin, A.H., et al. (1997) A novel five-transmembrane hematopoietic stem cell antigen: Isolation, characterization, and molecular cloning. Blood, 90, 5013-5021.

[12] Kobayashi, S.D., Voyich, J.M., Whitney, A.R. and DeLeo, F.R. (2005) Spontaneous neutrophil apoptosis and regulation of cell survival by granulocyte macrophage-colony stimulating factor. Journal of Leukocyte Biology, 78, 1408-1418. http://dx.doi.org/10.1189/jlb.0605289

[13] Vlkova, M., Fronkova, E., Kanderova, V., et al. (2010) Characterization of lymphocyte subsets in patients with common variable immunodeficiency reveals subsets of naive human B cells marked by CD24 expression. Journal of Immunology, 185, 6431-6438. http://dx.doi.org/10.4049/jimmunol.0903876

[14] Berga-Bolanos, R., Drews-Elger, K., Aramburu, J. and Lopez-Rodriguez, C. (2010) NFAT5 regulates T lymphocyte homeostasis and CD24-dependent $\mathrm{T}$ cell expansion under pathologic hypernatremia. Journal of Immunology, 185, 6624-6635. http://dx.doi.org/10.4049/jimmunol.1001232

[15] Jiang, W., Sui, X., Zhang, D., et al. (2011) CD24: A novel surface marker for PDX1-positive pancreatic progenitors derived from human embryonic stem cells. Stem Cells, 29, 609-607.

[16] Myung, J.H., Gajjar, K.A., Pearson, R.M., Launiere, C.A., Eddington, D.T. and Hong, S. (2011) Direct measurements on CD24-mediated rolling of human breast cancer MCF-7 cells on E-selectin. Analytical Chemistry, 83, 1078-1083. http://dx.doi.org/10.1021/ac102901e

[17] Romagnani, P. and Kalluri, R. (2009) Possible mechanisms of kidney repair. Fibrogenesis \& Tissue Repair, 2, 3. http://dx.doi.org/10.1186/1755-1536-2-3

[18] Ong, A.C. and Harris, P.C. (2005) Molecular pathogenesis of ADPKD: The polycystin complex gets complex. Kidney International, 67, 1234-1247. http://dx.doi.org/10.1111/j.1523-1755.2005.00201.x 